\title{
Timeliness of Materials on Reading Recommendation System
}

\author{
Yanling $\mathrm{Li}^{\# *}$, S.C. $\mathrm{Ng}^{*}$ \\ \#Department of Information Technology, Baoding University, Qiyi Road, Baoding, 071000, China \\ E-mail:67028728@qq.com \\ *School of Information Technology, SEGi University, Kota Damansara, Petaling Jaya, 47810, Malaysia \\ E-mail: ashleyng@segi.edu.my
}

\begin{abstract}
An improved fuzzy logic recommendation method named TFLRS is presented in this paper. The timeliness of reading materials is focused. The upload time of reading materials is attached as an important input parameter, and the numeric weights of input factors are further revised. The experiment result demonstrates that the recommendation ranking order of the latest and the out-of-date reading materials has obviously improved in comparison to the previous FLRS method. It solves the problem that the new reading materials cannot be timely discovered but the out-of-date reading materials always in the front of the recommendation ranking. The timeliness of reading materials effectively guarantees the user preferred newer materials are always at the higher level than the older materials in the recommendation ranking result and the accuracy of reading recommendation system has significantly improved.
\end{abstract}

Keywords - timeliness; recommendation method; fuzzy logic; fuzzy set; membership function

\section{INTRODUCTION}

As the increase of the reading materials for online learning, people feel more difficult to locate the content of their interest. Reading recommendation system for online learning is to timely, accurately and efficiently recommend different reading materials to relevant users based on different user preference [1]. A lot of methods were used in the previous study of recommendation system [2]. The most common methods are Collaborative Filtering algorithm, Content-Based algorithm and Hybrid algorithm [3]-[5]. These methods are successful in the practice of recommendation system, but they still remain some problems, such as cold start problem, data-sparse and some important factors of reading materials are not be effectively applied [6].

The author proposed a fuzzy logic method named FLRS on the reading recommendation system in previous research [7]. In this method, a fuzzy logic with numeric weight was proposed. Three input variables that user group preference, grade of reading materials and a click-through rate of reading materials was considered. Different numeric weights are attached to the input factors which affect recommendation ranking result of reading materials in FLRS. The experiment result demonstrated that FLRS method significantly improves the recommendation accuracy with $6.89 \%$ in comparison with the previous method. However, FLRS method does not consider the timeliness of reading materials. Some new reading materials that user preferred cannot be displayed in the front of the recommendation ranking result whereas some out-of-date reading materials in the front due to their high-grade feedback and high clickthrough rate. The reading materials residing in the front of the recommendation ranking result has higher click-through rate than those in the back. If the reading materials comments are good, the recommendation ranking order will be gradually stepping up to the front of the ranking. With the time going on, this results in some new reading materials may not have a chance to be clicked and read almost. As the result of being pushed down in the ranking order due to low exposure to users, these reading materials may disappear forever in the recommendation result.

This paper is an extended version of work published in [7]. In this study, an improved TFLRS method is proposed in which upload time factor of reading the material as a critical input parameter is considered in the design. The experimental results showed that the recommendation ranking order of the new and the old reading materials had improved significantly compared with the previous FLRS method.

Fuzzy logic method and timeliness of data are discussed in this research related works.

\section{A. Fuzzy Logic Method}

Fuzzy Logic is widely applied in vague and uncertain systems for it is more convenient to describe the input data, 
simulate human reasoning and interpret the output consequence [8]. Sinhal and Verma [9] proposed a fuzzy approach in the constructive cost model for the more precise effort estimation of the software development. It describes the detailed definition of each cost driver fuzzy set. The result proves that this method is better than the traditional methods. In another study, fuzzy logic was applied to automatic mining in cognitive metadata [10]. It introduced a metadata extraction framework based on the fuzzy inference. The documents were divided into semantic granules which were associated with fuzzy attributes and then inferred the possible metadata values. The results of the experiment showed that the application of fuzzy logic in this method had improved the precision, perceived quality and the performance on the metadata field. Wan et al. [11] presented an intelligent decision system for stock managers based on the fuzzy inference which can assist in deciding that people buy, sell or hold their stock. Fuzzy-based clustering was introduced in another study [12]. It categorized the web service's quality using a Fuzzy C-Means algorithm which assigned the data into different clusters with membership degree. Kumar et al. [13] compared the effectiveness of Linear Regression and five machine learning methods on soft testing time assessment model by training two failure data sets of real projects. Fuzzy inference system and adaptive neuro-fuzzy inference system are more beneficial in the assessment of software testing time. The adaptive neurofuzzy also was applied in web pages retrieval [14]. In this study, content page, page content of neighbours and link analysis were calculated, and the fuzzy system was applied to obtain the output.

There are some other studies introducing the application of fuzzy logic in the recommendation system [15], [16]. A fuzzy similarity matching model computed various similarity measures and translated it into fuzzy measurement scales to solve the problems of recommendation [17]. In addition, a numerical case study was demonstrated in this paper to prove the effectiveness and availability of the recommendation system. There is another recommendation framework with multi-agent based on fuzzy logic for elearning [18]. In the study, two fuzzy sets of input that user performance and user feedback were first defined, then it inferences the recommended study materials. It attempted to provide a personalized study environment based on learners' requirements, study goals and level. Chaudhari et al. [8] discussed a multilayer fuzzy inference system. The data weighted average was used in the first fuzzy layer, and the fuzzy output was applied in the second fuzzy layer. It provided a view in overcoming both types of the fuzzy system separately used. Muyeba et al. [19] proposed a fuzzy classification algorithm using fuzzy quantifiers in web mining. In the study, numeric weights were used in each traversed path based on the level of importance. The numeric weights can be calculated and translated into the fuzzy linguistic quantifiers attached to the fuzzy rules. From what has been discussed above, fuzzy logic is one of the advantage methods that can make the system behaving more intelligent and easier to describe the data used in linguistic variables like humans. It is more suitable to apply fuzzy logic to the situation in which the data is imprecise, uncertain and vague.

\section{B. Timeliness of Data}

The timeliness of data plays an important role in the network learning environment. The new data is more advanced and effective than older data for user [20]. So, more and more researchers begin to focus on the timeliness of data in their application. Luo et al. [20] proposed an algorithm that called timeliness online regularized extreme learning machine to improve the learning performance. In the study, a regularization technique for the timeliness scheme was added through applying a weight factor to achieve better generalization performance. The simulation results showed that this method achieved higher learning accuracy and better stability than other previous machine learning methods. Similarly, another machine learning algorithm which improves online sequential extreme learning based on timeliness management scheme was presented by $\mathrm{Gu}$ et al. [21]. The experimental result of the proposed method also outperformed other methods in higher learning accuracy and faster convergence. Han et al. [22] incorporated influence decay function, opportunistic selection and broader maximization accommodating to timeliness, acceptance ratio and breadth in their research titled an exploration of broader influence maximization in timeliness networks with the opportunistic selection. Kong et al. [23] also applied timeliness as an important factor to measure the efficiency of information acquisition in their research of optimizing social connections for efficient information acquisition. The characteristics that affect timeliness based on information seeking and organizational behaviour was identified by Ranganath et al. [24] to facilitate time-critical information seeking in social media. In another study, Shamsul and Dublin [25] focused the data quality changing with timeliness in information manufacturing system. Another timeliness optimization model based on information flow was established by Zhang et al. [26]. In the study, the timeliness optimization objectives and constraint condition were analysed, substructure bottleneck was iteratively optimized until the effect of optimization converge. Zhang et al. [27] focused a timeliness factor on improving the recommendation algorithms. In the result of the experiment, the obsolete items were suppressed strongly, and the recommendation accuracy was enhanced substantially. It can be summed up that all the research above mentioned are more beneficial in system performance after applied timeliness.

In this paper, the mean of timeliness is that the recommended trend and the distribution of the reading materials change with time passing by in reading recommendation system. The rest of this paper is organized as follows. Section 2 introduces fuzzy logic method design applied timeliness factor on reading materials system. In section 3, the experiment and results are analysed. Finally, a conclusion of this research will be pointed out in Section 4.

\section{MATERIAL AND METHOD}

\section{A. Fuzzy Set and Membership Function}

The fuzzy set can be defined with fuzzy boundaries. If $X$ is the reference super set discussed and FS is a subset of $\mathrm{X}$, then FS is a fuzzy subset of X, the definition of FS can be expressed by the following equation: 


$$
\mathrm{FS}=\left\{\left(x, \mu_{F S}(x)\right\} \quad x \in \mathrm{X}, \mu_{F S}(x): X \rightarrow[0,1]\right.
$$

where $\mu_{F S}(x)$ is the membership function of $x$.

The equation above can be extended to all the fuzzy subset FS limited reference super set $\mathrm{X}$ can be expressed as:

$$
\mathrm{FS}=\left\{\left(x_{1}, \mu_{F S}\left(x_{1}\right)\right\},\left\{\left(x_{2}, \mu_{F S}\left(x_{2}\right)\right\}, \ldots,\left\{\left(x_{n}, \mu_{F S}\left(x_{n}\right)\right\}\right.\right.\right.
$$

In this study, four influence factors of reading material recommendation as input variables. They are user group preference (UGP), grade of reading materials (GRM), a click-through rate of reading materials (CTR), upload time of reading materials(ULT). One output variable that recommendation degree (RM) is defined. The fuzzy set of this method can be expressed as follows:

$$
\begin{gathered}
F S_{\text {input }}=\left\{\left(x_{\mathrm{UGP}}, \mu_{U G P}\left(x_{\mathrm{UGP}}\right)\right\},\left\{\left(x_{G R M}, \mu_{G R M}\left(x_{\mathrm{GRM}}\right)\right\},\right.\right. \\
\left\{\left(x_{C T R}, \mu_{C T R}\left(x_{\mathrm{CTR}}\right)\right\},\left\{\left(x_{U L T}, \mu_{U T L}\left(x_{\mathrm{ULT}}\right)\right\}\right.\right. \\
F S_{\text {output }}=\left\{\left(x_{\mathrm{RM}}, \mu_{R M}\left(x_{\mathrm{RM}}\right)\right\}\right.
\end{gathered}
$$

All linguistic variables, linguistic values and their ranges are set up based on the domain expert. The fuzzy linguistic variables description of TFLRS method are shown in Table 1 to Table 5, and the relevant membership functions of these fuzzy sets are represented by Fig. 1 to Fig. 5.

TABLE I

UGP INPUT VARIABLE DETAILS

\begin{tabular}{|c|c|c|c|c|c|}
\hline Abbr. & VL & L & M & H & VH \\
\hline Describe & Very low & Low & Medium & High & Very high \\
\hline Range & $0-20$ & $10-40$ & $30-60$ & $50-80$ & $70-100$ \\
\hline
\end{tabular}

TABLE II

GRM INPUT VARIABLE DETAILS

\begin{tabular}{|c|c|c|c|c|c|}
\hline Abbr. & VB & B & F & G & VG \\
\hline Describe & Very bad & Bad & Fair & Good & Very good \\
\hline Range & $0-20$ & $10-40$ & $30-60$ & $50-80$ & $70-100$ \\
\hline
\end{tabular}

TABLE III

CTR INPUT VARIABLE DETAILS

\begin{tabular}{|c|c|c|c|c|c|}
\hline Abbr. & VL & L & M & H & VH \\
\hline Describe & Very low & Low & Medium & High & Very high \\
\hline Range & $0-20$ & $10-40$ & $30-60$ & $50-80$ & $70-100$ \\
\hline
\end{tabular}

TABLE IV

ULT INPUT VARIABLE DETAILS

\begin{tabular}{|c|c|c|c|c|c|}
\hline Abbr. & VL & L & G & N & VN \\
\hline Describe & Very low & Low & General & New & Very new \\
\hline Range & $0-20$ & $10-40$ & $30-70$ & $60-90$ & $80-100$ \\
\hline
\end{tabular}

TABLE V

RM OUTPUT VARIABLE DETAILS

\begin{tabular}{|c|c|c|c|c|}
\hline Abbr. & NR & LR & R & HR \\
\hline Describe & $\begin{array}{c}\text { No } \\
\text { recommend }\end{array}$ & $\begin{array}{c}\text { Low } \\
\text { recommend }\end{array}$ & recommend & $\begin{array}{c}\text { High } \\
\text { recommend }\end{array}$ \\
\hline Range & $0-30$ & $10-50$ & $40-80$ & $70-100$ \\
\hline
\end{tabular}

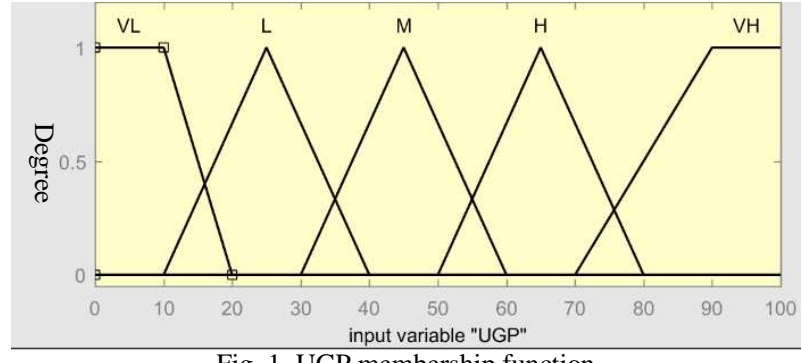

Fig. 1 UGP membership function

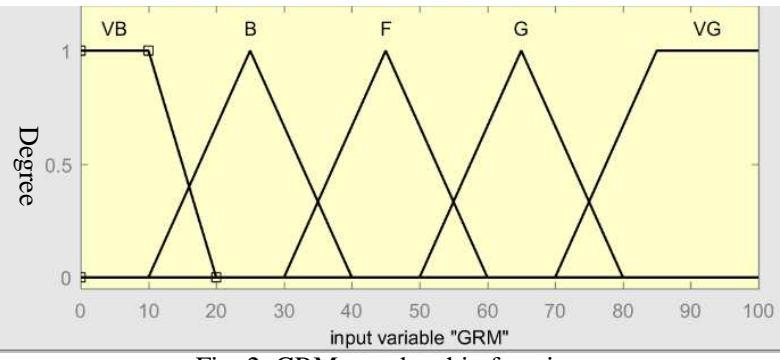

Fig. 2 GRM membership function

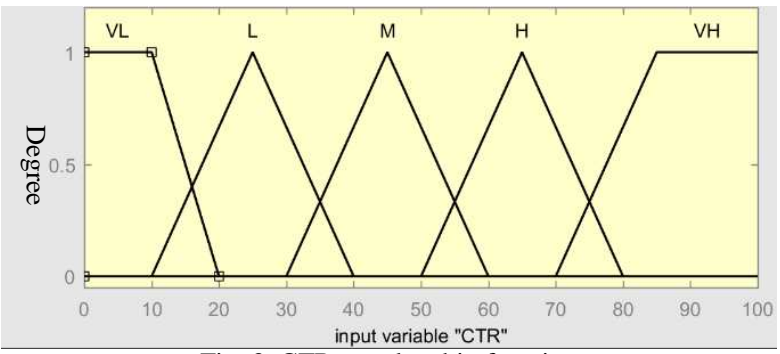

Fig. 3 CTR membership function

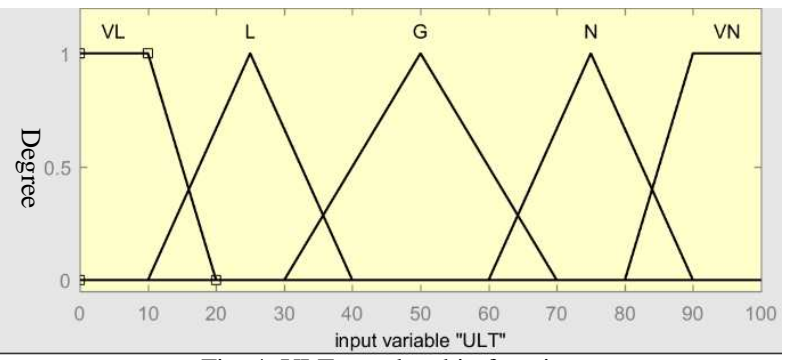

Fig. 4 ULT membership function

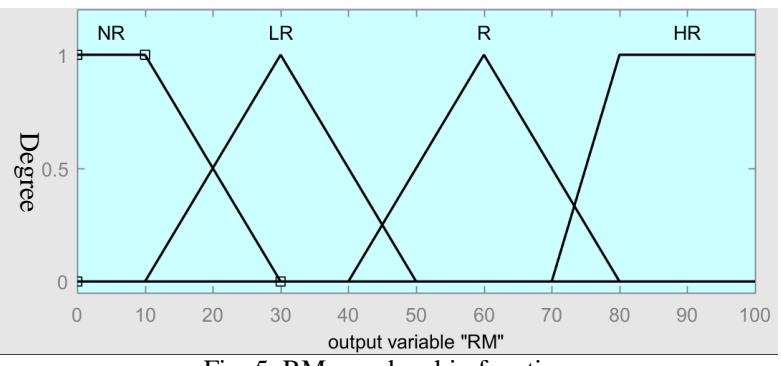

Fig. 5 RM membership function

Because the important degree of input variables is different based on the real practice, in this study, a set of weight is used in the input parameters.

$$
\mathrm{W}=\left\{\mathrm{w}_{1}, \mathrm{w} 2, \ldots, \mathrm{w}_{\mathrm{i}}, \ldots, \mathrm{w}_{\mathrm{n}}\right\} \mathrm{w}_{\mathrm{i}} \in[0,1] \text { and } \sum_{1}^{\mathrm{n}} \mathrm{w}_{\mathrm{i}}=1
$$


The new membership degree is calculated as:

$$
y_{i}=w_{i} * \mu_{F S}\left(x_{i}\right)
$$

where $\mu_{F S}\left(x_{i}\right)$ is the membership function of $\mathrm{x}_{\mathrm{i}}, w_{i}$ is the weight of the membership function and $y_{i}$ is the ultimate membership degree result.

\section{B. Fuzzy Rules}

The general form of fuzzy rules that multiple inputs and one output variables are expressed as follows:

If ( $\mathrm{x}_{1}$ is $\mathrm{FS}_{1} \mid$ not $\mathrm{FS}_{1}$ [and $\mathrm{x}_{2}$ is $\mathrm{FS}_{2} \mid$ not FS2 and... and $\mathrm{x}_{\mathrm{n}}$ is $\mathrm{FS}_{\mathrm{n}} \mid$ not $\mathrm{FS}_{\mathrm{n}}$ ]) then ( $\mathrm{y}$ is FR).

If $\left(\mathrm{x}_{1}\right.$ is $\mathrm{FS}_{1} \mid \operatorname{not} \mathrm{FS}_{1}\left[\right.$ or $\mathrm{x}_{2}$ is $\mathrm{FS}_{2} \mid \operatorname{not} \mathrm{FS} 2$ or... or $\mathrm{x}_{\mathrm{n}}$ is $\mathrm{FS}_{\mathrm{n}} \mid$ not $\mathrm{FS}_{\mathrm{n}}$ ]) then ( $\mathrm{y}$ is FR).

where $x_{n}$ is the value of input parameters, $F_{n}$ is the fuzzy set of input parameters, and FR is the fuzzy set of the output parameter. The fuzzy rules of TFLRS method are represented in Fig. 6 and the rule viewer in the system shown in Fig. 7.

\footnotetext{
1. If (UPG is VL) then (RM is NR) (1)

2. If (UPG is $L$ ) and (ULT is VL) then (RM is NR) (1)

3. If (UPG is $L$ ) and (ULT is $L$ ) then (RM is NR) (1)

4. If (UPG is $L$ ) and (ULT is $G$ ) then (RM is NR) (1)

5. If (UPG is $L$ ) and (GRM is VB) and (ULT is N) then (RM is NR) (1)

6. If (UPG is $L$ ) and (GRM is $B$ ) and (ULT is N) then (RM is NR) (1)

7. If (UPG is $L$ ) and (GRM is F) and (ULT is N) then (RM is LR) (1)

8. If (UPG is $L$ ) and (GRM is $G$ ) and (ULT is N) then (RM is $L R$ ) (1)

9. If (UPG is L) and (GRM is VG) and (ULT is N) then (RM is LR) (1)

10. If (UPG is $L$ ) and (GRM is VB) and (CTR is VL) and (ULT is VN) then (RM is LR) (1)

11. If (UPG is $L$ ) and (GRM is VB) and (CTR is L) and (ULT is VN) then (RM is LR) (1)

12. If (UPG is $L$ ) and (GRM is VB) and (CTR is M) and (ULT is VN) then (RM is LR) (1)

13. If (UPG is $L$ ) and (GRM is VB) and (CTR is H) and (ULT is VN) then (RM is NR) (1)

14. If (UPG is $L$ ) and (GRM is VB) and (CTR is VH) and (ULT is VN) then (RM is NR) (1)

15. If (UPG is $M$ ) and (ULT is VL) then (RM is NR) (1)

16. If (UPG is $M$ ) and (ULT is L) then (RM is NR) (1)

17. If (UPG is M) and (GRM is VB) and (ULT is G) then (RM is NR) (1)

18. If (UPG is M) and (GRM is not VB) and (ULT is G) then (RM is LR) (1)

19. If (UPG is M) and (GRM is VB) and (CTR is VL) and (ULT is N) then (RM is LR) (1)

20. If (UPG is M) and (GRM is VB) and (CTR is $L$ ) and (ULT is N) then (RM is LR) (1)

21. If (UPG is M) and (GRM is VB) and (CTR is M) and (ULT is N) then (RM is LR) (1)

22. If (UPG is M) and (GRM is VB) and (CTR is H) and (ULT is N) then (RM is NR) (1)

23. If (UPG is M) and (GRM is VB) and (CTR is VH) and (ULT is N) then (RM is NR) (1)

24. If (UPG is M) and (GRM is $B$ ) and (CTR is VL) and (ULT is N) then (RM is LR) (1)

25. If (UPG is M) and (GRM is $B$ ) and (CTR is L) and (ULT is N) then (RM is LR) (1)

26. If (UPG is $M$ ) and (GRM is $B$ ) and (CTR is $M$ ) and (ULT is N) then (RM is $R$ ) (1)

27. If (UPG is $M$ ) and (GRM is $B$ ) and (CTR is $H$ ) and (ULT is $N$ ) then (RM is $R$ ) (1)

29. If (UPG is $M$ ) and (GRM is F) and (ULT is N) then (RM is $R$ ) (1)

29. If (UPG is $M$ ) and (GRM is $G$ ) and (ULT is $N$ ) then (RM is $R$ ) (1)

31. If (UPG is M) and (GRM is VG) and (ULT is N) then (RM is $R$ ) (1)

32. If (UPG is $M$ ) and (GRM is VB) and (ULT is VN) then (RM is $L R$ ) (1)

33. If (UPG is $M$ ) and (GRM is not VB) and (ULT is VN) then (RM is R) (1)

34. If (UPG is $H$ ) and (GRM is VB) and (CTR is VL) and (ULT is VL) then (RM is LR) (1)

36. If (UPG is $H$ ) and (GRM is VB) and (CTR is M) and (ULT is VL) then (RM is NR) (1)

37. If (UPG is $H$ ) and (GRM is VB) and (CTR is $H$ ) and (UTT is VL) then (RM is NR) (1)

38. If (UPG is $H$ ) and (GRM is VB) and (CTR is VH) and (ULT is VL) then (RM is NR) (1)

39. If (UPG is $H$ ) and (GRM is not VB) and (ULT is VL) then (RM is LR) (1)

40. If (UPG is $H$ ) and (GRM is VB) and (CTR is VL) and (ULT is L) then (RM is LR) (1)

42. If (UPG is $H$ ) and (GRM is VB) and (CTR is M) and (ULT is L) then (RM is LR) (1)

43. If (UPG is $H$ ) and (GRM is VB) and (CTR is $H$ ) and (ULT is L) then (RM is NR) (1)

44. If (UPG is $H$ ) and (GRM is VB) and (CTR is VH) and (ULT is L) then (RM is NR) (1)

45. If (UPG is H) and (GRM is not VB) and (ULT is L) then (RM is LR) (1)

46. If (UPG is H) and (GRM is VB) and (CTR is VL) and (ULT is G) then (RM is R) (1)

47. If (UPG is $H$ ) and (GRM is VB) and (CTR is $L$ ) and (ULT is $G$ ) then (RM is R) (1)

48. If (UPG is $H$ ) and (GRM is VB) and (CTR is M) and (ULT is $G$ ) then (RM is LR) (1)

49. If (UPG is $H$ ) and (GRM is VB) and (CTR is $H$ ) and (ULT is G) then (RM is LR) (1)

50. If (UPG is $H$ ) and (GRM is VB) and (CTR is VH) and (ULT is G) then (RM is LR) (1)

51. If (UPG is $H$ ) and (GRM is not VB) and (CTR is VH) and (ULT is G) then (RM is R) (1)

52. If (UPG is $H$ ) and (GRM is VB) and (CTR is VL) and (ULT is N) then (RM is HR) (1)

53. If (UPG is H) and (GRM is VB) and (CTR is L) and (ULT is N) then (RM is HR) (1)

54. If (UPG is $H$ ) and (GRM is VB) and (CTR is M) and (ULT is N) then (RM is R) (1)

55. If (UPG is H) and (GRM is VB) and (CTR is H) and (ULT is N) then (RM is LR) (1)

56. If (UPG is H) and (GRM is VB) and (CTR is VH) and (ULT is N) then (RM is LR) (1)

57. If (UPG is H) and (GRM is not VB) and (ULT is N) then (RM is HR) (1)

58. If (UPG is H) and (GRM is VB) and (CTR is VL) and (ULT is VN) then (RM is HR) (1)

59. If (UPG is $H)$ and (GRM is VB) and (CTR is $L$ ) and (ULT is VN) then (RM is HR) (1)

60. If (UPG is $H$ ) and (GRM is VB) and (CTR is $M$ ) and (ULT is VN) then (RM is R) (1)

62. If (UPG is $H$ ) and (GRM is VB) and (CTR is $H$ ) and (ULT is VN) then (RM is R) ( 1 )

63. If (UPG is $H$ ) and (GRM is not VB) and (ULT is VN) then (RM is HR) (1)

64. If (UPG is $V H$ ) and (GRM is VB) and (CTR is VL) and (UTT is VL) then (RM is $L R$ ) (1)

65. If (UPG is $V H$ ) and (GRM i $V$ ) and (CTR is $L$ ) and (ULT is VL) (hen (RM is LR) (1)

66. If (UPG is VH) and (GRM i VB) and (CTR is) and (ULT is VL) then (RM is NR) (1)

67. If (UPG is VH) and (GRM is VB) and (CTR is $H$ ) and (ULT is VL) then (RM is NR) (1)

68. If (UPG is VH) and (GRM is VB) and (CTR is VH) and (ULT is VL) then (RM is NR) (1)
}

69. If (UPG is VH) and (GRM is not VB) and (ULT is VL) then (RM is LR) (1)

70. If (UPG is VH) and (GRM is VB) and (CTR is VL) and (ULT is $L$ ) then (RM is LR) (1) 71. If (UPG is VH) and (GRM is VB) and (CTR is $L$ ) and (ULT is $L$ ) then (RM is $L R$ ) (1) 72 If (UPG is VH) and (GRM is VB) and (CTR is $M$ ) and (ULT is $L$ ) then (RM is $L R$ ) (1) 73. If (UPG is VH) and (GRM is VB) and (CTR is $H$ ) and (ULT is $L$ ) then (RM is NR) (1) 74. If (UPG is VH) and (GRM is VB) and (CTR is VH) and (ULT is L) then (RM is NR) (1) 75. If (UPG is VH) and (GRM is not VB) and (ULT is L) then (RM is $L R$ ) (1) 76. If (UPG is VH) and (GRM is VB) and (CTR is VL) and (ULT is G) then (RM is R) (1) 77. If (UPG is VH) and (GRM is VB) and (CTR is $L$ ) and (ULT is $G$ ) then (RM is $R$ ) (1) 78. If (UPG is VH) and (GRM is VB) and (CTR is M) and (ULT is G) then (RM is LR) (1) 79. If (UPG is VH) and (GRM is VB) and (CTR is H) and (ULT is G) then (RM is LR) (1) 80. If (UPG is VH) and (GRM is VB) and (CTR is VH) and (ULT is G) then (RM is LR) (1) 81. If (UPG is VH) and (GRM is not VB) and (ULT is G) then (RM is R) (1)

82. If (UPG is VH) and (GRM is VB) and (CTR is VL) and (ULT is N) then (RM is HR) (1) 83. If (UPG is VH) and (GRM is VB) and (CTR is L) and (ULT is N) then (RM is HR) (1) 84. If (UPG is VH) and (GRM is VB) and (CTR is M) and (ULT is N) then (RM is R) (1) 85. If (UPG is VH) and (GRM is $\vee B$ ) and (CTR is $H$ ) and (ULT is $N$ ) then (RM is LR) (1) 86. If (UPG is VH) and (GRM is VB) and (CTR is VH) and (ULT is N) then (RM is LR) (1) 87. If (UPG is VH) and (GRM is not VB) and (ULT is N) then (RM is HR) (1) 88. If (UPG is VH) and (GRM is VB) and (CTR is VL) and (ULT is VN) then (RM is HR) (1) 89. If ( $U P G$ is $V H$ ) and (GRM is VB) and (CTR is $L$ ) and ( $U L T$ is VN) then (RM is HR) (1) 90. If (UPG is VH) and (GRM is VB) and (CTR is M) and (ULT is VN) then (RM is R) (1) 91. If (UPG is VH) and (GRM is VB) and (CTR is H) and (ULT is VN) then (RM is R) (1) 92. If (UPG is VH) and (GRM is VB) and (CTR is VH) and (ULT is VN) then (RM is R) (1)
93. If (UPG is VH) and (GRM is not VB) and (ULT is VN) then (RM is HR) (1)

Fig. 6 Fuzzy rules

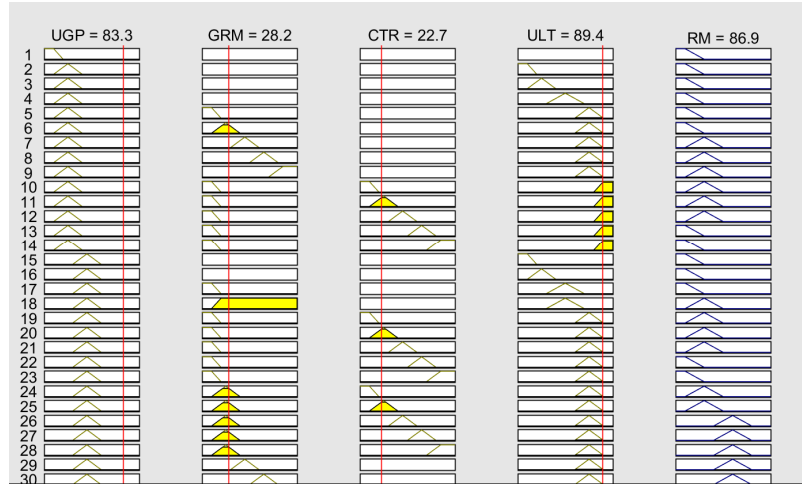

Fig. 7 Rule viewer TFLRS

\section{Defuzzification}

Defuzzification is to translate the fuzzy output into a crisp number. There are a lot of methods for defuzzification, such as Centroid, Bisector and the Mean of Maximum (MOM). In this study, a centroid method is applied for defuzzification. The mathematical equation of centroid can be expressed as following.

$$
\mathrm{C}=\frac{\sum_{x=a}^{b} y_{i} x_{i}}{\sum_{x=a}^{b} y_{i}}
$$

where $y_{i}$ is the membership degree of $x_{i}, \mathrm{a}, \mathrm{b}$ is the interval values of output fuzzy set. $\mathrm{C}$ is the crisp output value of defuzzification. The 3D surface of defuzzification with ULT and other parameters shown in Fig. 8 -Fig. 10:

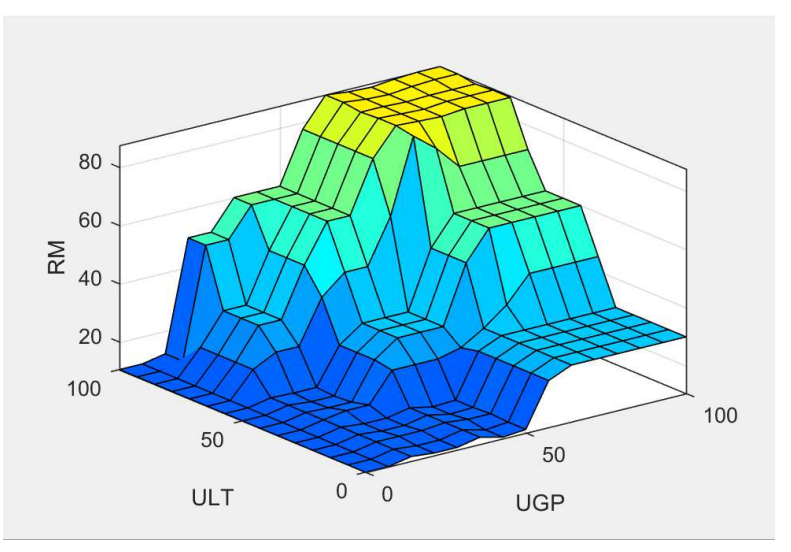

Fig. 8 3D defuzzification surface with ULT and UGP 


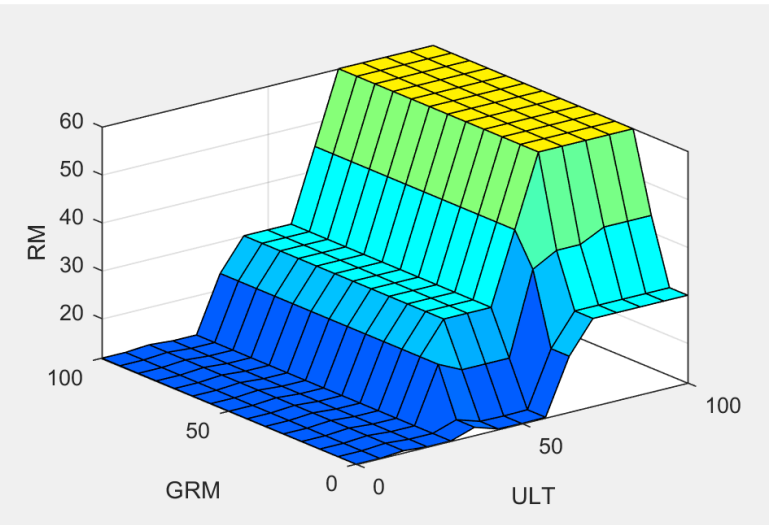

Fig. 9 3D defuzzification surface with GRM and ULT

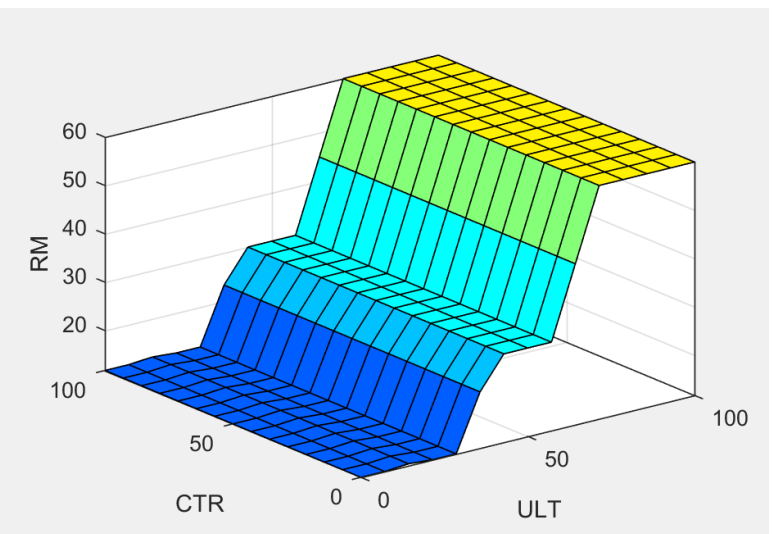

Fig. 10 3D defuzzification surface with CTR and ULT

TABLE VI

FLRS INPUT VALUES

\begin{tabular}{|c|c|c|c|}
\hline $\begin{array}{c}\text { Input } \\
\text { Number }\end{array}$ & UGP & GRM & CTR \\
\hline 1 & 99.66212 & 83.12475 & 91.32184 \\
\hline 2 & 94.04104 & 13.74342 & 16.36572 \\
\hline 3 & 91.28758 & 26.10445 & 38.45479 \\
\hline 4 & 88.13761 & 95.96448 & 77.67326 \\
\hline 5 & 85.92308 & 30.03728 & 21.12382 \\
\hline 6 & 83.41861 & 72.72388 & 86.83671 \\
\hline 7 & 79.44624 & 90.95055 & 84.92698 \\
\hline 8 & 74.72919 & 29.3384 & 33.22551 \\
\hline 9 & 82.52748 & 29.50114 & 12.06081 \\
\hline 10 & 87.15758 & 98.09146 & 71.34621 \\
\hline 11 & 86.80514 & 11.15762 & 5.736578 \\
\hline 12 & 83.34611 & 88.33933 & 75.31729 \\
\hline 13 & 76.76616 & 90.01953 & 98.47112 \\
\hline 14 & 83.27332 & 21.50748 & 28.24741 \\
\hline 15 & 81.24802 & 34.50693 & 22.59507 \\
\hline
\end{tabular}

\section{RESULTS AND DISCUSSION}

In the experiment, 100 reading materials was selected to recommend to users. Among the 100 reading materials, 15 special reading materials of user preference was observed: 8 reading materials upload time are new but the comment grade and click-through rate are lower, the other 7 reading materials upload time are long while the comment grade and click-through rate are higher.

\section{A. FLRS Method}

There are three input variables in FLRS: UGP, GRM and CTR. The input values of 15 reading materials as following Table 6.

The numerical weights of input variables are set as following:

$$
w_{i}=\left\{\begin{array}{ll}
0.4 & U G P \\
0.4 & G R M \\
0.2 & C T R
\end{array} \quad i=1,2,3\right.
$$

The defuzzification output value $\mathrm{RM}$ and the ranking order show in Table 7.

\section{B. TFLRS Method}

ULT parameter was added in this method. Because the comment grade is null for the new materials, the weight of GRM was reduced. This study suggests that it is more accurate to enhance the important degree of ULT and reduces the important degree of GRM in real situation.

TABLE VII

FLRS OUTPUT RESULTS

\begin{tabular}{|c|c|c|}
\hline Number & RM & Ranking \\
\hline 1 & 85.4954 & 1 \\
\hline 2 & 14.3444 & 87 \\
\hline 3 & 47.0161 & 46 \\
\hline 4 & 85.3889 & 11 \\
\hline 5 & 59.9855 & 39 \\
\hline 6 & 85.4954 & 2 \\
\hline 7 & 85.4925 & 6 \\
\hline 8 & 43.5939 & 47 \\
\hline 9 & 58.0247 & 44 \\
\hline 10 & 85.37 & 12 \\
\hline 11 & 14.0225 & 97 \\
\hline 12 & 85.4826 & 8 \\
\hline 13 & 85.4313 & 10 \\
\hline 14 & 29.5296 & 64 \\
\hline 15 & 59.9639 & 40 \\
\hline
\end{tabular}

The numerical weights of input variables are revised as following:

$$
w_{i}=\left\{\begin{array}{cc}
0.3 & \text { UGP } \\
0.2 & \text { GRM } \\
0.2 & \text { CTR } \\
0.3 & \text { ULT }
\end{array} \quad i=1,2,3,4\right.
$$

The ULT input value, RM output value and ranking order show in Table 8.

TABLE VIII

TFLRS ULT INPUT VALUES AND RESULTS

\begin{tabular}{|c|c|c|c|}
\hline Number & ULT & RM & Ranking \\
\hline 1 & 3.4746 & 30.0256 & 52 \\
\hline 2 & 96.7342 & 85.3441 & 5 \\
\hline 3 & 85.2437 & 85.1697 & 13 \\
\hline 4 & 23.6148 & 30.0093 & 59 \\
\hline 5 & 88.9438 & 85.2053 & 11 \\
\hline 6 & 18.5693 & 30.0176 & 56 \\
\hline 7 & 24.6543 & 30.0149 & 58 \\
\hline 8 & 88.4672 & 85.2678 & 9 \\
\hline 9 & 76.8759 & 85.3242 & 6 \\
\hline 10 & 12.6418 & 30.0025 & 62 \\
\hline 11 & 88.4352 & 85.459 & 2 \\
\hline
\end{tabular}




\begin{tabular}{|l|l|l|l|}
\hline 12 & 24.5324 & 30.0081 & 60 \\
\hline 13 & 36.2547 & 46.8021 & 39 \\
\hline 14 & 93.2459 & 85.1875 & 12 \\
\hline 15 & 82.5631 & 85.1274 & 15 \\
\hline
\end{tabular}

\section{Discussion}

The experiment results of recommendation ranking order in TFLRS method are compared with FLRS method in Fig. 11. In fig, 'o' is devote the ranking order result of FLRS method and ' $*$ ' is devote the ranking order result of TFLRS method. The ranking order and difference value is show in Table 9.

The experiment results showed that all the 15 reading materials recommendation ranking order have greater change. The greatest ranking change is the 11 th reading material. This reading material has very bad user comment and very low click-through rate, but because the materials upload time is very new, the recommendation ranking order up from the 97th to the 2nd place. Meanwhile, the 6th reading material has good user comment and very high click-through rate, but the upload time is very long, so the ranking order down from the 2 nd to the 56th. In additional, the least change of recommendation ranking order is the 15 th reading material, it also adjust 25 ranking orders.

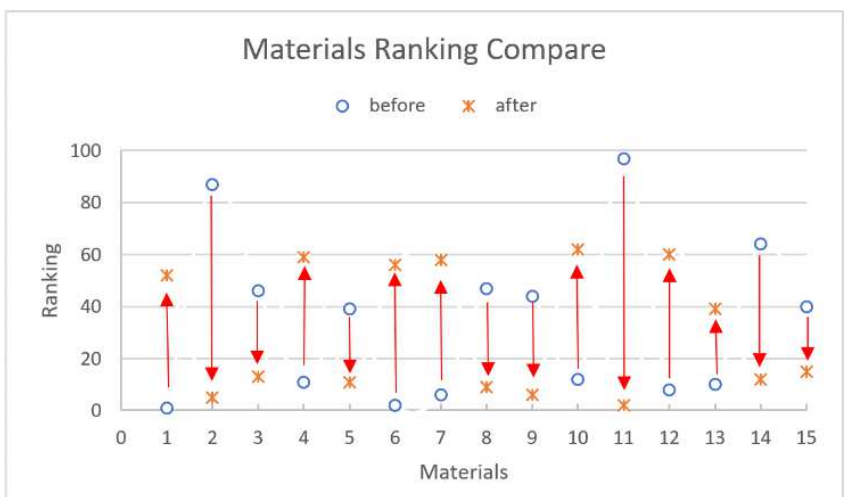

Fig. 11 Ranking order in TFLRS and FLRS

TABLE IX

RANKING ORDER AND DIFFERENCE VALUE

\begin{tabular}{|c|c|c|c|}
\hline Number & FLRS & TFLRS & Deference value \\
\hline 1 & 1 & 52 & 51 \\
\hline 2 & 87 & 5 & 82 \\
\hline 3 & 46 & 13 & 33 \\
\hline 4 & 11 & 59 & 48 \\
\hline 5 & 39 & 11 & 28 \\
\hline 6 & 2 & 56 & 54 \\
\hline 7 & 6 & 58 & 52 \\
\hline 8 & 47 & 9 & 38 \\
\hline 9 & 44 & 6 & 38 \\
\hline 10 & 12 & 62 & 50 \\
\hline 11 & 97 & 2 & 95 \\
\hline 12 & 8 & 60 & 52 \\
\hline 13 & 10 & 39 & 29 \\
\hline 14 & 64 & 12 & 52 \\
\hline 15 & 40 & 15 & 25 \\
\hline
\end{tabular}

To verify the consistency of the experiment, another two groups reading materials were selected and repeated this experiment. The results of experiment also showed that the latest reading materials can display at the further front of the recommendation ranking order and the out-of-date reading materials were at the further back orders. The comparison of ranking order between TFLRS method and FLRS method for the three times experiments is illustrated by the Fig. 12 .

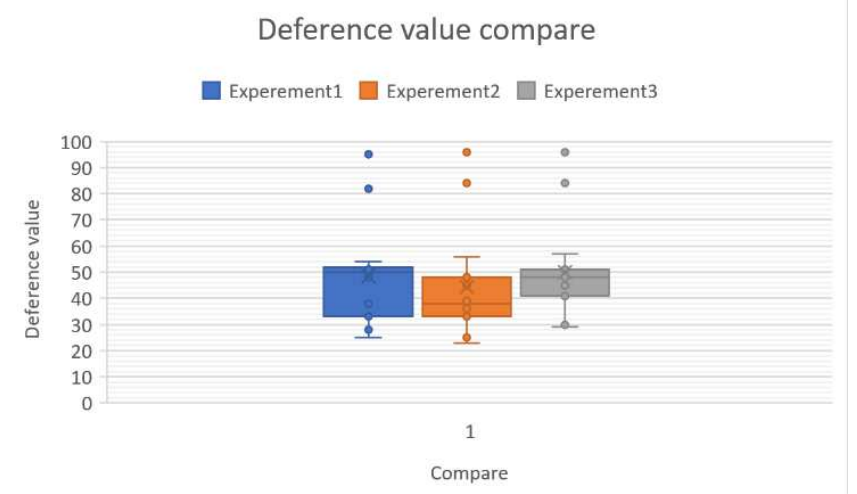

Fig. 12 The deference values between TFLRS and FLLRS in three times experiments

The experiment results show that the ranking order have improved with $48.47 \%, 44.4 \%$ and $50.07 \%$ in three times experiments respectively and the average ranking order has been improved with $47.64 \%$. The outcome shows that it is obviously revise the order of recommendation materials after applied ULT parameter in TFLRS method. It is better than FLRS method in the timeliness of reading materials.

\section{CONCLUSION}

In this study, an improved fuzzy logic method that focuses on timeliness of reading materials was introduced. The ULT parameter as an important input variable is attached and the numeric weight of input parameters based on their important degree has been revised in practicing reading recommendation system. The experiment result demonstrates that the proposed method successful adjusts the recommendation ranking order of the latest and out-ofdate reading materials. It enables the new reading materials of user preference are always at the further front of the recommendation sequence. The accuracy of recommendation results obviously outdistances FLRS method. Future research includes but is not limited to the following topics: how to dynamically adjust the weights associated with the parameters and a Neuro Network approach will be used in the system.

\section{REFERENCES}

[1] J. Lian, "Research on User Features Based Daa Minging in Social Networks," 2013.

[2] J. Lu, D. Wu, M. Mao, W. Wang, and G. Zhang, "Recommender system application developments: A survey," Decis. Support Syst., vol. 74, pp. 12-32, 2015.

[3] M. Salehi, I. Nakhai Kamalabadi, and M. B. Ghaznavi Ghoushchi, "Personalized recommendation of learning material using sequential pattern mining and attribute based collaborative filtering," Educ. Inf. Technol., pp. 1-23, 2012.

[4] A. Elkahky, "A Multi-View Deep Learning Approach for Cross Domain User Modeling in Recommendation Systems," pp. 278-288, 2015.

[5] J. N. K. Liu and V. W. S. Cho, "A Hybrid Algorithm for Recommendation Twitter Peers *," pp. 644-649, 2014.

[6] J. Bian, B. Long, L. Li, T. Moon, A. Dong, and Y. Chang, "Exploiting User Preference for Online Learning in Web Content Optimization Systems," Yichang-Cs.Com, vol. V, no. 212, pp. 1-23, 2014. 
[7] Y. Li et al., "Design of a Reading Recommendation Method Based on User Preference for Online Learning," no. 4, pp. 519-522, 2015.

[8] S. Chaudhari, M. Patil, and J. Bambhori, "Study and Review of Fuzzy Inference Systems for Decision Making and Control," Am. Int. J. Res. Sci. Technol. Eng. Math., pp. 88-92, 2014.

[9] A. Sinhal and B. Verma, "A novel fuzzy based approach for effort estimation in software development," ACM SIGSOFT Softw. Eng. Notes, vol. 38, no. 5, p. 1, 2013.

[10] M. Sah and V. Wade, "Automatic mining of cognitive metadata using fuzzy inference," Proc. 22nd ACM Conf. Hypertext hypermedia - HT' '11, p. 37, 2011.

[11] H. Wan and G. W. Kuriger, "an Intelligent Decision Support System for," pp. 52-55, 2011

[12] M. H. Hasan, J. Jaafar, and M. F. Hassan, "Fuzzy-based Clustering of Web Services' Quality of Service: A Review," J. Commun., vol. 9, no. 1, pp. 81-90, 2014.

[13] P. Kumar and Y. Singh, "Assessment of Software Testing Time Using Soft Computing Techniques," SIGSOFT Softw. Eng. Notes, vol. 37, no. 1, pp. 1-6, 2012.

[14] M. S. Iraji, H. Maghamnia, and M. Iraji, "Web Pages Retrieval with Adaptive Neuro Fuzzy System based on Content and Structure," Int. J. Mod. Educ. Comput. Sci., vol. 7, no. 8, pp. 69-84, 2015.

[15] D. Wu, J. Lu, and G. Zhang, "A Fuzzy Tree Matching-based Personalized e-Learning Recommender System," IEEE Trans. Fuzzy Syst., vol. 6706, no. c, pp. 1-1, 2014.

[16] S.-M. Chen, S.-H. Cheng, and T.-E. Lin, "Group decision making systems using group recommendations based on interval fuzzy preference relations and consistency matrices," Inf. Sci. (Ny)., vol. 298, pp. 555-567, 2015.

[17] K. Lin and C. Chiu, "A Fuzzy Similarity Matching Model for Interior Design Drawing Recommendation,” vol. 1, pp. 2-7, 2015.
[18] H. Pandey, "A Fuzzy Logic based Recommender System for ELearning System with Multi-Agent Framework," vol. 122, no. 17, pp. 18-21, 2015.

[19] M. K. Muyeba and L. Han, "Fuzzy classification in web usage mining using fuzzy quantifiers," Proc. 2013 IEEE/ACM Int. Conf. Adv. Soc. Networks Anal. Min. - ASONAM '13, pp. 1381-1386, 2013.

[20] X. Luo, X. Yang, C. Jiang, and X. Ban, "Timeliness online regularized extreme learning machine," Int. J. Mach. Learn. Cybern., 2016.

[21] Y. Gu, J. Liu, Y. Chen, X. Jiang, and H. Yu, "Neurocomputing TOSELM : Timeliness Online Sequential Extreme Learning Machine," Neurocomputing, vol. 128, pp. 119-127, 2014.

[22] M. Han, M. Yan, Z. Cai, and Y. Li, "Journal of Network and Computer Applications An exploration of broader in $\mathrm{fl}$ uence maximization in timeliness networks with opportunistic selection," J. Netw. Comput. Appl., vol. 63, pp. 39-49, 2016.

[23] C. Kong, "Optimizing Social Connections for Efficient Information Acquisition," 2016.

[24] S. Ranganath, S. Wang, X. Hu, J. Tang, and H. Liu, "Seeking in Social Media," vol. 29, no. 10, pp. 2197-2209, 2017.

[25] M. S. Islam, "An Assessment For Focusing The Change Of Data Quality ( DQ ) With Timeliness In Information Manufacturing System ( IMS ), 2013.

[26] Z. Jinfeng, Y. Kan, W. Heng, M. Shaojie, and Z. Jieyong, "Timeliness Optimization Model For Network C 4 ISR System Structure Based on Information Flow," no. Asei, pp. 745-749, 2015.

[27] F. Zhang, Q. Liu, and A. Zeng, "Timeliness in recommender systems," Expert Syst. Appl., vol. 85, pp. 270-278, 2017. 